\title{
Partitions sans petites parts
}

\author{
par EliE MOSAKI, JEAN-Louis NICOLAS et ANDRÁS SÁRKÖZY
}

\begin{abstract}
RÉSUMÉ. On désigne par $r(n, m)$ le nombre de partitions de l'entier $n$ en parts supérieures ou égales à $m$. En partant de l'estimation asymptotique de $r(n, m)$ exprimée à l'aide d'un paramètre $\sigma$ défini implicitement en fonction de $n$ et $m$, nous éliminons ce paramètre en utilisant la formule sommatoire d'Euler-Maclaurin, pour obtenir un développement asymptotique de $r(n, m)$ valable pour $n \rightarrow+\infty$, et $1 \leq m \leq \Gamma \sqrt{n}, \Gamma$ étant un réel quelconque.
\end{abstract}

ABSTRACT. Let $r(n, m)$ denote the number of partitions of $n$ into parts, each of which is at least $m$. Starting from the asymptotic estimate of $r(n, m)$ which use a parameter $\sigma$ implicitely defined in terms of $m$ and $n$, we eliminate this parameter by using the EulerMaclaurin formula, and obtain an asymptotics for $r(n, m)$ in terms of $m$ and $n$ only, which holds for $n \rightarrow+\infty$, and $1 \leq m \leq \Gamma \sqrt{n}$, where $\Gamma$ is a given real.

\section{Introduction}

On désignera par $\mathbb{N}$ l'ensemble des entiers positifs ou nuls, et $\mathbb{N}^{*}$ l'ensemble des entiers strictement positifs.

Soit $p(n)$ le nombre de partitions de l'entier naturel $n$, c'est-à-dire,

$$
p(n)=\left|\left\{\left(i_{1}, \ldots, i_{r}\right) \in \mathbb{N}^{r} ; n=i_{1}+\cdots+i_{r}, 1 \leq i_{1} \leq \cdots \leq i_{r}, r \in \mathbb{N}^{*}\right\}\right| .
$$

Hardy et Ramanujan (voir [4]) ont étudié le comportement asymptotique de $p(n)$ quand $n$ tend vers l'infini, et on a par exemple

$$
p(n)=\frac{e^{2 c \sqrt{n}}}{4 \sqrt{3} n}\left[1+O\left(\frac{1}{\sqrt{n}}\right)\right] \text { où } c=\frac{\pi}{\sqrt{6}}=1,2825 \ldots
$$

Dans tout cet article, la constante $c$ aura la valeur donnée ci-dessus. On définit respectivement $r(n, m)$ le nombre de partitions de l'entier naturel $n$ en parts supérieures ou égales à $m$, et $q(n, m)$ le nombre de partitions de

Manuscrit reçu le 23 juin 2003.

Recherche partiellement financée par le CNRS, Institut Girard Desargues, UMR 5028, et the Hungarian National Fondation for Scientific Research, grant No. TO43623. 
l'entier naturel $n$ en parts distinctes supérieures ou égales à $m, m$ étant un réel supérieur ou égal à 1 , c'est-à-dire,

$r(n, m)=\left|\left\{\left(i_{1}, \ldots, i_{r}\right) \in \mathbb{N}^{r} ; n=i_{1}+\cdots+i_{r}, m \leq i_{1} \leq \cdots \leq i_{r}, r \in \mathbb{N}^{*}\right\}\right|$,

$q(n, m)=\left|\left\{\left(i_{1}, \ldots, i_{r}\right) \in \mathbb{N}^{r} ; n=i_{1}+\cdots+i_{r}, m \leq i_{1}<\cdots<i_{r}, r \in \mathbb{N}^{*}\right\}\right|$.

Le but du présent article est de donner un développement asymptotique de $r(n, m)$ uniformément en $m, 1 \leq m \leq \Gamma n^{1 / 2}, \Gamma$ étant un réel fixé. Le résultat de base est le théorème Nicolas-Sárközy (voir [9]) dont nous donnons une version un peu modifiée qui améliore sensiblement l'estimation $\mathrm{du}$ reste (pour les détails voir [8]), ce reste $E$ (voir ci-après) demeurant maintenant le même au voisinage de $m=\lambda \sqrt{n}$, et étant valable pour des valeurs de $m$ encore plus proches de $n$ :

Théorème 1.1. $\Pi$ existe des réels $d(x, y),(x, y) \in \mathbb{N}^{2}, y \in[1, x]$, tel que, si $L_{x}=\sum_{i=1}^{x} d(x, i) A(x, i)$ ò̀ $A(h, \ell)=\sum_{j=m}^{n} \frac{j^{h}}{\left(e^{\sigma j}-1\right)^{\ell}}$, alors, pour tout $k \in \mathbb{N}, k \geq 3$, quand $n \rightarrow+\infty$, on $a$

$$
r(n, m)=\frac{1}{\sqrt{\pi} B} e^{\sigma n} \prod_{j=m}^{n} \frac{1}{1-e^{-\sigma j}} Q
$$

où $\sigma$ et $B$ sont donnés par

$$
B^{2}=L_{2}=2 A(2,1)+2 A(2,2)=2 \sum_{j=m}^{n} \frac{j^{2} e^{\sigma j}}{\left(e^{\sigma j}-1\right)^{2}}
$$

et

$$
Q=1+\sum_{2 \leq i \leq(3 k-6) / 2}(-1)^{i} Q_{2 i}+E
$$

avec

$$
Q_{2 i}=2^{-i}(2 i-1)(2 i-3) \cdot \ldots \cdot 1 \cdot L_{2}^{-i} \sum_{\max \left\{1, \frac{2 i-k+2}{2}\right\} \leq t \leq k-2} \frac{1}{t !} \cdot \sum_{\substack{h_{1}+h_{2}+\ldots+h_{t}=2 i \\ 3 \leq h_{1}, \ldots, h_{t} \leq k}} L_{h_{1}} L_{h_{2}} \ldots L_{h_{t}}
$$

et pour tout $\Gamma$ réel assez grand,

$$
E \ll \Gamma \begin{cases}n^{-(k-1) / 4}(\log n)^{3(k-1) / 2} & \text { si } m \leq \Gamma n^{1 / 2} \\ \left(\frac{m}{n}\right)^{(k-1) / 2}(\log n)^{3(k-1) / 2} & \text { si } m>\Gamma n^{1 / 2}\end{cases}
$$


ceci uniformément pour $m$ tel que $1 \leq m \leq \frac{n}{(\log n)^{3}} \varepsilon(n)$, ò̀ $\varepsilon$ désigne n'importe quelle suite tendant vers 0 quand $n$ tend vers $+\infty$.

L'analogue pour la fonction $q(n, m)$ est le théorème de Freiman et Pitman (voir [3]) :

quand $n \rightarrow+\infty$, on a

$$
q(n, m)=\frac{1}{\left(2 \pi B^{2}\right)^{1 / 2}} e^{\sigma n} \prod_{j=m}^{n}\left(1+e^{-\sigma j}\right)(1+E)
$$

où $\sigma$ et $B$ sont donnés par

$$
n=\sum_{j=m}^{n} \frac{j}{1+e^{\sigma j}}
$$

et

$$
B^{2}=\sum_{j=m}^{n} \frac{j^{2} e^{\sigma j}}{\left(1+e^{\sigma j}\right)^{2}}
$$

et

$$
E=E(m, n)=O\left((\log n)^{9 / 2} \max \left\{n^{-1 / 4},(m / n)^{1 / 2}\right\}\right)
$$

uniformément pour $m$ tel que

$$
1 \leq m \leq \frac{K_{0} n}{(\log (n))^{9}} .
$$

Ici $K_{0}$ et les constantes implicites dans l'estimation de $E$ sont des constantes positives indépendantes de $m$ et $n$.

Les résultats que nous obtiendrons amélioreront ceux obtenus par Dixmier-Nicolas en 1987 (voir [2]) : uniformément pour $1 \leq m \leq n^{1 / 4}$,

$$
r(n, m)=p(n)\left(\frac{\pi}{\sqrt{6 n}}\right)^{m-1}(m-1) !\left[1+O\left(m^{2} / \sqrt{n}\right)\right]
$$

et par Herzog dans sa thèse en 1987 (voir [6]) : si $m=O\left(n^{3 / 8}(\log n)^{1 / 4}\right)$,

(1.6) $\log r(n, m)=2 c \sqrt{n}-\frac{1}{2} m \log n+m \log m-m(1-\log c)$

$$
+O\left(n^{1 / 4} \sqrt{\log n}\right) .
$$

Ils généraliseront également les résultats obtenus par Dixmier-Nicolas en 1990 (voir [1]) :

- si $m=\lambda \sqrt{n}, \lambda>0$,

$$
\log r(n, m) \sim g(\lambda) \sqrt{n} \text { quand } n \rightarrow+\infty
$$


où $g$ est une fonction particulière dont nous rappellerons les principales propriétés un peu plus loin dans l'article.

- si $m \leq n^{1 / 3-\varepsilon}$,

$$
r(n, m) \sim p(n)\left(\frac{\pi}{\sqrt{6 n}}\right)^{m-1}(m-1) ! \exp \left[-\frac{1}{4}\left(c+\frac{1}{c}\right) \frac{m^{2}}{\sqrt{n}}\right] .
$$

A partir du théorème de Nicolas-Sárközy, nous allons exprimer $r(n, m)$ en fonction de $n$ et $m$ en éliminant le paramètre $\sigma$.

Nous étudierons simplement le cas $m=O\left(n^{1 / 2}\right)$; le cas $\Gamma n^{1 / 2} \leq m \leq n$, avec $\Gamma$ réel positif assez grand, sera examiné dans un article ultérieur.

La méthode que nous employons ici pourrait s'appliquer également à la fonction $q(n, m)$ étudiée par Freiman et Pitman, et tous leurs résultats peuvent être retrouvés et approfondis.

Enonçons le théorème principal :

Théorème 1.2. Soit $\Gamma \geq 2$, réel aussi grand que l'on veut. Pour tout $\ell \in \mathbb{N}$, on a uniformément pour $m \leq \Gamma n^{1 / 2}$,

$$
\begin{aligned}
& r(n, m)=\frac{e^{2 c \sqrt{n}}}{4 \sqrt{3} n}\left(\frac{\pi}{\sqrt{6 n}}\right)^{m-1}(m-1) ! \exp \left[\sqrt{n} \tilde{g}(\lambda)+g_{0}(\lambda)+\right. \\
&\left.+\cdots+\frac{1}{n^{\frac{\ell}{2}}} g_{\ell}(\lambda)+O\left(\frac{1}{n^{\frac{\ell+1}{2}}}\right)\right]
\end{aligned}
$$

où $\lambda=\frac{m}{\sqrt{n}}$, et $\tilde{g}, g_{0}, \ldots, g_{\ell}$ sont analytiques sur $\mathbb{R}^{+}$.

De façon plus précise pour $\tilde{g}$ et $g_{0}$, on a pour tout $x$ positif ou nul,

$$
\begin{aligned}
\tilde{g}(x) & =g(x)-(x \log x+2 c+(\log c-1) x) \\
& =2\left(\frac{H(x)}{x}-c+\frac{x}{2}\right)+x \log \left(\frac{1-e^{-H(x)}}{c x}\right)
\end{aligned}
$$

et

$$
\begin{aligned}
g_{0}(x)= & \log \left(\frac{H(x)}{c x}\right) \\
- & \frac{1}{2} \log \left(\frac{1-e^{-H(x)}}{H(x)}\right) \\
& -\frac{1}{2} \log \left(1-\frac{3}{\pi^{2}} \int_{0}^{H(x)} \frac{t^{2} e^{t}}{\left(e^{t}-1\right)^{2}} d t\right) \\
= & \frac{H(x)}{2}-\frac{1}{2} \log \left(1+\frac{e^{H(x)}-1-H(x)+\frac{x^{2}}{2}}{H(x)}\right)
\end{aligned}
$$

où les fonctions $g$ et $H$ seront définies au paragraphe $\mathbf{2}$.

En particulier, si $x$ tend vers 0 , on obtient des développements limités à tout ordre de $\tilde{g}$ et $g_{0}$, qu'on écrira plus loin dans l'article. 
Nous démontrerons au paragraphe 3 le théorème 1.2 en appliquant le théorème 1.1 pour $\frac{k-1}{4}>\frac{\ell+1}{2}$, soit $k>1+2(\ell+1)=2 \ell+3 ; k=2 \ell+4$ conviendra.

L'outil principal pour la démonstration du théorème 1.2 est la formule sommatoire d'Euler-Maclaurin qui va nous permettre d'évaluer $\sigma$, donné par (1.2), à partir de l'intégrale $\int_{m}^{n} \frac{t}{e^{\sigma t}-1} d t$, et l'utilisation de la formule de Taylor.

L'utilisation de Maple a été une source de vérification efficace pour tous nos calculs.

\section{Quelques fonctions utiles}

Avant de démontrer le théorème 1.2, nous allons introduire certaines fonctions utiles, déjà étudiées par G. Szekeres (voir [10] et [11]).

2.1. Soit, pour $x$ réel,

$$
F(x)=\int_{x}^{\infty} \frac{t}{e^{t}-1} d t
$$

On a, pour $x \geq 0$,

$$
F(x)=\int_{x}^{\infty} t\left(\sum_{n=1}^{\infty} e^{-n t}\right) d t=\sum_{n=1}^{\infty} \int_{x}^{\infty} t e^{-n t} d t=\sum_{n=1}^{\infty}\left(\frac{x}{n}+\frac{1}{n^{2}}\right) e^{-n x}
$$

et en particulier,

$$
F(0)=\sum_{n=1}^{\infty} \frac{1}{n^{2}}=\frac{\pi^{2}}{6}=c^{2} .
$$

D'autre part, par (2.1) et (2.3),

$$
F(x)=\frac{\pi^{2}}{6}-\int_{0}^{x} \frac{t}{e^{t}-1} d t
$$

En utilisant le fait que $t \mapsto \frac{t}{e^{t}-1}-\frac{t}{2}$ est une fonction paire, on obtient pour $x$ réel,

$$
F(-x)=\frac{x^{2}}{2}+\frac{\pi^{2}}{3}-F(x)
$$

La fonction $F$ est analytique sur $\mathbb{R}$ et strictement décroissante de $+\infty$ à 0 . On connait le développement en série entière de $t \mapsto \frac{t}{e^{t}-1}$ défini à l'aide des nombres de Bernoulli $B_{n}$ qui seront introduits en 3.1 et on obtient alors celui de $F$ en utilisant la formule (2.4) :

$$
F(x)=\frac{\pi^{2}}{6}-\sum_{n \geq 0} \frac{B_{n}}{(n+1) !} x^{n+1}
$$


On a par exemple :

$$
F(x)=\frac{\pi^{2}}{6}-x+\frac{x^{2}}{4}-\frac{x^{3}}{36}+\frac{x^{5}}{3600}+O\left(x^{7}\right) \quad \text { quand } x \rightarrow 0 .
$$

Lorsque $x \rightarrow+\infty$, on obtient avec (2.2),

$$
F(x)=(x+1) e^{-x}+O\left(x e^{-2 x}\right) .
$$

2.2. On pose alors pour $x$ réel,

$$
G(x)=\frac{x}{\sqrt{F(x)}}
$$

La fonction $G$ est analytique sur $\mathbb{R}$, et

$$
\forall x \in \mathbb{R} \quad, \quad G^{\prime}(x)=\frac{F(x)+\frac{x^{2}}{2\left(e^{x}-1\right)}}{F(x)^{3 / 2}}
$$

soit, à l'aide d'une intégration par parties dans la définition (2.1) de $F$,

$$
\forall x \in \mathbb{R} \quad, \quad G^{\prime}(x)=\frac{1}{2} \frac{\int_{x}^{\infty} \frac{t^{2} e^{t}}{\left(e^{t}-1\right)^{2}} d t}{F(x)^{3 / 2}}>0 .
$$

Or à l'aide de la définition (2.8) de $G$ et de (2.5), on a

$$
\lim _{x \rightarrow-\infty} G(x)=-\sqrt{2}
$$

et de plus

$$
\lim _{x \rightarrow+\infty} G(x)=+\infty
$$

donc $G$ réalise une bijection croissante de $\mathbb{R}$ dans $]-\sqrt{2},+\infty[$.

Un développement limité de $F$ en 0 fournit un développement limité de $G$ en 0 et l'on a par exemple le développement limité de $G$ à l'ordre 4 en 0 :

$$
G(x)=\frac{1}{c} x+\frac{1}{2 c^{3}} x^{2}+\frac{-c^{2}+3}{8 c^{5}} x^{3}+\frac{2 c^{4}-27 c^{2}+45}{144 c^{7}} x^{4}+O\left(x^{5}\right) .
$$

Tous les coefficients de ce développement limité sont des fractions rationnelles en $c$.

2.3. On peut alors définir $H=G^{-1}$. $H$ est strictement croissante de ]$-\sqrt{2},+\infty[$ sur $\mathbb{R}$ et analytique sur $]-\sqrt{2},+\infty[$. On obtient par un procédé classique d'inversion (cf Knuth [7], §4.7), par exemple re-injections 
dans (2.9), un développement limité en 0 de $H$ :

$$
\begin{aligned}
& \text { (2.10) } H(x)=c x-\frac{x^{2}}{2}+\frac{1}{8}\left(c+\frac{1}{c}\right) x^{3}-\frac{1}{72}\left(c^{2}+9\right) x^{4} \\
& +\frac{1}{384} \frac{17 c^{4}+18 c^{2}-3}{c^{3}} x^{5}+\frac{1}{7200}\left(c^{4}-50 c^{2}-325\right) x^{6} \\
& +\frac{1}{414720} \frac{56 c^{8}+7425 c^{6}+7875 c^{4}-2025 c^{2}+405}{c^{5}} x^{7}+O\left(x^{8}\right)
\end{aligned}
$$

A nouveau, tous les coefficients de ce développement limité sont des fractions rationnelles en $c$.

De plus si $x>-\sqrt{2}, H(x)$ vérifie

$$
\left(\frac{H(x)}{x}\right)^{2}=F(H(x))=\int_{H(x)}^{\infty} \frac{t}{e^{t}-1} d t
$$

(en appliquant $G(y)=\frac{y}{\sqrt{F(y)}}$ à $\left.y=H(x)\right)$.

2.4. Pour $\lambda>0$, posons

$$
g(\lambda)=2 \frac{H(\lambda)}{\lambda}+\lambda \log \left(1-e^{-H(\lambda)}\right)
$$

On donne une autre expression de $g$ qui nous sera utile par la suite; pour cela, effectuons une intégration par parties dans (2.11) pour obtenir

$$
\left(\frac{H(\lambda)}{\lambda}\right)^{2}=-H(\lambda) \log \left(1-e^{-H(\lambda)}\right)-\int_{H(\lambda)}^{\infty} \log \left(1-e^{-t}\right) d t
$$

et finalement, en reprenant (2.12),

$$
g(\lambda)=\frac{H(\lambda)}{\lambda}-\frac{\lambda}{H(\lambda)} \int_{H(\lambda)}^{\infty} \log \left(1-e^{-t}\right) d t
$$

Rappelons que $H$ est analytique en 0 , et s'annule en 0 (en considérant (2.10) par exemple). En mettant (2.12) sous la forme

$$
g(\lambda)=\lambda \log \lambda+2 \frac{H(\lambda)}{\lambda}+\lambda \log \left(\frac{1-e^{-H(\lambda)}}{H(\lambda)}\right)+\lambda \log \left(\frac{H(\lambda)}{\lambda}\right)
$$


on obtient que $\lambda \mapsto g(\lambda)-\lambda \log \lambda$ est analytique en 0 , donc de la forme $\sum_{n \geq 0} a_{n} \lambda^{n}$. Et on a, en utilisant (2.10),

$$
\begin{cases}a_{0}=2 c & a_{4}=-\frac{1}{576} \frac{17 c^{4}+18 c^{2}-3}{c^{3}} \\ a_{1}=\log c-1 & a_{5}=-\frac{1}{14400}\left(c^{4}-50 c^{2}-325\right) \\ a_{2}=-\frac{1}{4}\left(c+\frac{1}{c}\right) & a_{6}=-\frac{56 c^{8}+7425 c^{6}+7875 c^{4}-2025 c^{2}+405}{1036800 c^{5}} \\ a_{3}=\frac{c^{2}+9}{72} & \ldots\end{cases}
$$

les $a_{i}$ étant des fractions rationnelles en $c=\frac{\pi}{\sqrt{6}}(i \geq 2)$.

Proposition 2.1. La fonction g définie par (2.12) coïncide avec la fonction $g$ de la formule (1.7) : si $m=\lambda \sqrt{n}, \lambda>0$,

$$
\log r(n, m) \sim g(\lambda) \sqrt{n} \text { quand } n \rightarrow+\infty \text {. }
$$

Démonstration. D'après la preuve du théorème 2.15 de [1], il suffit de montrer que $g$ vérifie les conditions suivantes :

(i) $g(\lambda) \rightarrow a_{0}>0$ quand $\lambda \rightarrow 0$,

(ii) $g^{\prime}(\lambda) \rightarrow-\infty$ quand $\lambda \rightarrow 0$,

(iii) $\lambda g^{\prime}(\lambda) \rightarrow 0$ quand $\lambda \rightarrow 0$

(iv) $\lambda^{2} g^{\prime \prime}+\lambda g^{\prime}-g=\frac{2 g^{\prime \prime}}{1-\exp \left(-g^{\prime}\right)}$.

Les trois conditions (i), (ii), (iii) s'obtiennent facilement puisque quand $\lambda \rightarrow 0, \lambda \log \lambda \rightarrow 0$ et $g^{\prime}(\lambda)=\log \lambda+1+\sum_{n \geq 1} n a_{n} \lambda^{n-1}$.

Il nous reste à démontrer (iv) : pour $\lambda>0$, on a :

$$
g^{\prime}(\lambda)=2 \frac{H^{\prime}(\lambda)}{\lambda}-2 \frac{H(\lambda)}{\lambda^{2}}+\frac{\lambda H^{\prime}(\lambda) e^{-H(\lambda)}}{1-e^{-H(\lambda)}}+\log \left(1-e^{-H(\lambda)}\right) .
$$

Or, par (2.11), on obtient, si $x>0$ :

$$
2 H^{\prime}(x) H(x)=2 x\left(\frac{H(x)}{x}\right)^{2}+x^{2} \frac{H^{\prime}(x) H(x)}{1-e^{H(x)}}
$$

donc,

$$
2 H^{\prime}(x)=2 \frac{H(x)}{x}+x^{2} \frac{H^{\prime}(x)}{1-e^{H(x)}}
$$

donc,

$$
x \frac{H^{\prime}(x) e^{-H(x)}}{1-e^{-H(x)}}=2 \frac{H(x)}{x^{2}}-2 \frac{H^{\prime}(x)}{x} .
$$


Ainsi

$$
g^{\prime}(\lambda)=\log \left(1-e^{-H(\lambda)}\right)
$$

D'où

$$
g^{\prime \prime}(\lambda)=\frac{H^{\prime}(\lambda) e^{-H(\lambda)}}{1-e^{-H(\lambda)}}=\frac{H^{\prime}(\lambda)}{e^{H(\lambda)}-1}
$$

et finalement

$$
\lambda^{2} g^{\prime \prime}(\lambda)+\lambda g^{\prime}(\lambda)-g(\lambda)=\lambda^{2} \frac{H^{\prime}(\lambda)}{e^{H(\lambda)}-1}-2 \frac{H(\lambda)}{\lambda}=-2 H^{\prime}(\lambda)
$$

or

$$
1-e^{-g^{\prime}(\lambda)}=1-\frac{1}{1-e^{-H(\lambda)}}=\frac{1}{1-e^{H(\lambda)}}
$$

donc

$$
\frac{2 g^{\prime \prime}(\lambda)}{1-\exp \left(-g^{\prime}(\lambda)\right)}=-2 H^{\prime}(\lambda)
$$

Ainsi (iv) est démontré.

Remarque. La ressemblance des coefficients $a_{i}$ de $g$ en (2.14) avec ceux de $H$ en (2.10) provient de la formule :

$$
\frac{d}{d \lambda}\left(\frac{g(\lambda)}{\lambda}\right)=-2 \frac{H(\lambda)}{\lambda^{3}}
$$

qui résulte de la relation $(2.15)$ et de $(2.12)$.

\section{Démonstration du théorème 1.2}

La formule (1.1) du théorème 1.1 donne

(3.1) $\log r(n, m)=-\frac{1}{2} \log \pi-\log B+\sigma n+\sum_{j=m}^{n}-\log \left(1-e^{-\sigma j}\right)+\log Q$

Lorsque nous aurons évalué $\sigma$ de façon précise en fonction de $n$ et $m$ à l'aide de la formule (1.2) et de la formule sommatoire d'Euler-Maclaurin (que nous rappelons dans la proposition 3.1 ci-dessous), nous pourrons évaluer de façon précise également, toujours à l'aide de la formule sommatoire d'EulerMaclaurin, $-\log B$, défini par (1.3), $\sum_{j=m}^{n}-\log \left(1-e^{-\sigma j}\right)$ et $\log Q$, défini par (1.4). Nous pourrons alors écrire le développement asymptotique de $r(n, m)$ en fonction de $n$ et $m$ uniquement. 


\subsection{Formule sommatoire d'Euler-Maclaurin.}

Rappelons deux définitions équivalentes des polynômes de Bernoulli $\left(B_{n}(x)\right)$ (voir [5] chapitre XIII) :

$$
e^{z x} \frac{z}{e^{z}-1}=\sum_{n \geq 0} \frac{B_{n}(x)}{n !} z^{n} \quad \text { ou } \quad\left\{\begin{array}{l}
B_{n}(1)=B_{n}(0), n \geq 2 \\
B_{n}^{\prime}=n B_{n-1}, n \geq 1 \\
B_{0}=1
\end{array}\right.
$$

On note alors $B_{i}=B_{i}(0)$ les nombres de Bernoulli. On a

$$
\begin{gathered}
B_{0}=1, \quad B_{1}=-\frac{1}{2}, \quad B_{2}=\frac{1}{6}, B_{3}=0, B_{4}=-\frac{1}{30}, \quad B_{5}=0, \ldots \\
B_{0}(x)=1, \quad B_{1}(x)=x-\frac{1}{2}, \quad B_{2}(x)=x^{2}-x+\frac{1}{6}, \ldots
\end{gathered}
$$

Proposition 3.1. (Formule sommatoire d'Euler-Maclaurin, voir [5] chapitre XIII).

Soit $k \in \mathbb{N}^{*},(p, q) \in \mathbb{N}^{2}$ tels que $q>p$, et $f: \mathbb{R}^{+} \rightarrow \mathbb{R}$ telle que $\int_{p}^{q}\left|f^{(2 k)}\right|<\infty$, alors

$\sum_{j=p}^{q} f(j)=\int_{p}^{q} f+\frac{1}{2}[f(p)+f(q)]+\sum_{j=1}^{k-1} \frac{B_{2 j}}{(2 j) !}\left[f^{(2 j-1)}(q)-f^{(2 j-1)}(p)\right]+R_{p, q}^{f}(k)$

où

$$
R_{p, q}^{f}(k)=\int_{p}^{q} \frac{B_{2 k}-B_{2 k}(x-\lfloor x\rfloor)}{(2 k) !} f^{(2 k)}(x) d x
$$

On a

$$
\left|R_{p, q}^{f}(k)\right| \leq \frac{4}{(2 \pi)^{2 k}} \int_{p}^{q}\left|f^{(2 k)}\right| .
$$

\subsection{Première estimation de $\sigma$.}

Lemme 3.1. Pour tout $m$ et $n$, $\sigma$ défini par (1.2) vérifie $\sigma \leq \frac{\pi}{\sqrt{6 n}}$ et si $m \leq \Gamma \sqrt{n}$ et $n \geq 4 \Gamma^{2}$, alors $\sigma \geq \frac{\log \Gamma}{\Gamma \sqrt{n}}$.

Ce lemme fournit un ordre de grandeur de $\sigma$, qui sera amélioré par la suite.

Démonstration. Notons d'abord que $\sigma$ est strictement positif et déterminé de façon unique puisque la fonction $t \mapsto \sum_{j=m}^{n} \frac{j}{t^{j}-1}$ est décroissante sur ] $1,+\infty[$ et a comme limites $+\infty$ et 0 en 1 et $+\infty$ respectivement; donc $n$ a un unique antécédent par cette fonction sur $] 1,+\infty[$, qui se met de façon unique sous la forme $e^{\sigma}$ avec $\sigma$ strictement positif . 
La fonction $t \mapsto \frac{t}{e^{t \sigma}-1}$ étant positive et décroissante sur $\mathbb{R}^{+}$, on a d'après $(1.2),(2.1)$ et $(2.3)$ :

$$
n=\sum_{j=m}^{n} \frac{j}{e^{\sigma j}-1} \leq \int_{m-1}^{n} \frac{t}{e^{\sigma t}-1} d t \leq \int_{0}^{\infty} \frac{t}{e^{\sigma t}-1} d t=\frac{1}{\sigma^{2}} F(0)=\frac{\pi^{2}}{6 \sigma^{2}}
$$

d'où,

$$
\sigma \leq \frac{\pi}{\sqrt{6 n}}
$$

Pour la deuxième partie de la preuve, prenons $r=\Gamma \sqrt{n}$ si bien que $[r, 2 r] \subset$ $[m, n]$, alors

$$
\begin{aligned}
n=\sum_{j=m}^{n} \frac{j}{e^{\sigma j}-1} \geq \int_{m}^{n} \frac{t}{e^{t \sigma}-1} & d t \geq \int_{r}^{2 r} \frac{t}{e^{t \sigma}-1} d t \\
& \geq \int_{r}^{2 r} \frac{t}{e^{t \sigma}} d t \geq e^{-2 r \sigma} \int_{r}^{2 r} t d t \geq r^{2} e^{-2 r \sigma},
\end{aligned}
$$

d'où

$$
\sigma \geq \frac{1}{2 r} \log \frac{r^{2}}{n}=\frac{1}{r} \log \frac{r}{\sqrt{n}}=\frac{\log \Gamma}{\Gamma \sqrt{n}}
$$

3.3. La formule sommatoire d'Euler-Maclaurin pour $A(h, \ell)$.

Rappelons que $A(h, \ell)$, introduit dans le théorème 1.1, est défini pour $(h, \ell) \in \mathbb{N}^{2}$, par

$$
A(h, \ell)=\sum_{j=m}^{n} \frac{j^{h}}{\left(e^{\sigma j}-1\right)^{\ell}} .
$$

Lemme 3.2. Soit $m \leq \Gamma \sqrt{n}$, alors pour tout $(h, \ell) \in \mathbb{N}^{2}, 1 \leq \ell \leq h$, et pour tout $k \geq 1$, on $a$

$$
\begin{aligned}
A(h, \ell)=\frac{1}{\sigma^{h+1}}\left[\int_{\sigma m}^{+\infty} U+\frac{\sigma}{2} U(\sigma m)\right. & \\
& \left.-\sum_{j=1}^{k-1} \frac{B_{2 j}}{(2 j) !} \sigma^{2 j} U^{(2 j-1)}(\sigma m)+O\left(\frac{1}{n^{k}}\right)\right]
\end{aligned}
$$

ò̀ $U(t)=U(h, \ell, t)=\frac{t^{h}}{\left(e^{t}-1\right)^{\ell}}$ si $t \in \mathbb{R}$.

Démonstration. Appliquons la formule sommatoire d'Euler-Maclaurin (proposition 3.1) à l'ordre $k$ à la fonction $U_{\sigma}=U \circ \varphi$ où $\varphi(t)=\sigma t$, sur l'intervalle 
$[m, n]$; c'est légitime puisque $U$ est analytique sur $\mathbb{R}$ ( elle l'est en 0 car $h \geq \ell): \forall k \geq 1$,

$$
\begin{aligned}
A(h, \ell)= & \sum_{j=m}^{n} \frac{j^{h}}{\left(e^{\sigma j}-1\right)^{\ell}}=\frac{1}{\sigma^{h}} \sum_{j=m}^{n} U_{\sigma}(j) \\
= & \frac{1}{\sigma^{h}}\left[\int_{m}^{n} U_{\sigma}+\frac{1}{2}\left[U_{\sigma}(m)+U_{\sigma}(n)\right]\right. \\
& \left.\quad+\sum_{j=1}^{k-1} \frac{B_{2 j}}{(2 j) !}\left[U_{\sigma}^{(2 j-1)}(n)-U_{\sigma}^{(2 j-1)}(m)\right]+R_{m, n}^{U_{\sigma}}(k)\right] .
\end{aligned}
$$

Simplifions cette expression, sachant que $\forall \ell \in \mathbb{N}, U_{\sigma}^{(\ell)}=\sigma^{\ell} \times U^{(\ell)} \circ \varphi$;

$$
\begin{aligned}
A(h, \ell)= & \frac{1}{\sigma^{h+1}}\left[\int_{\sigma m}^{\sigma n} U+\frac{\sigma}{2}[U(\sigma m)+U(\sigma n)]\right. \\
& \left.+\sum_{j=1}^{k-1} \frac{B_{2 j}}{(2 j) !} \sigma^{2 j}\left[U^{(2 j-1)}(\sigma n)-U^{(2 j-1)}(\sigma m)\right]+\sigma R_{m, n}^{U_{\sigma}}(k)\right] \\
= & \frac{1}{\sigma^{h+1}}\left[\int_{\sigma m}^{+\infty} U+\frac{\sigma}{2} U(\sigma m)-\sum_{j=1}^{k-1} \frac{B_{2 j}}{(2 j) !} \sigma^{2 j} U^{(2 j-1)}(\sigma m)+\tilde{R}\right]
\end{aligned}
$$

où

$$
\tilde{R}=-\int_{\sigma n}^{+\infty} U+\frac{\sigma}{2} U(\sigma n)+\sum_{j=1}^{k-1} \frac{B_{2 j}}{(2 j) !} \sigma^{2 j} U^{(2 j-1)}(\sigma n)+\sigma R_{m, n}^{U_{\sigma}}(k) .
$$

Il reste à démontrer que

$$
\tilde{R}=O\left(\frac{1}{n^{k}}\right) .
$$

D'après le lemme 3.1, $\sigma n$ tend vers $+\infty$ quand $n$ tend vers $+\infty$, et le comportement de $U$ et de ses dérivées en $+\infty$ va être l'argument principal pour conclure. On a

$$
\forall j \geq 0, U^{(j)}(t) \sim(-1)^{j} \ell^{j} t^{h} e^{-\ell t} \text { quand } t \rightarrow+\infty .
$$

Pour cela, il suffit de montrer que pour tout $t>0$,

$$
U^{(j)}(t)=(-1)^{j} \sum_{r \geq 0}\left(\begin{array}{c}
r+\ell-1 \\
\ell-1
\end{array}\right) \sum_{s=0}^{\min (h, j)}(-1)^{s}\left(\begin{array}{l}
j \\
s
\end{array}\right) \frac{h !}{(h-s) !}(r+\ell)^{j-s} t^{h-s} e^{-(r+\ell) t}
$$


ce qui se prouve en dérivant par la formule de Leibnitz, après avoir écrit $U$ sous la forme

$$
\begin{aligned}
U(t)=\frac{t^{h}}{\left(e^{t}-1\right)^{\ell}}=\frac{t^{h} e^{-\ell t}}{\left(1-e^{-t}\right)^{\ell}} & =t^{h} e^{-\ell t} \sum_{r \geq 0}\left(\begin{array}{c}
r+\ell-1 \\
\ell-1
\end{array}\right)\left(e^{-t}\right)^{r} \\
& =\sum_{r \geq 0}\left(\begin{array}{c}
r+\ell-1 \\
\ell-1
\end{array}\right) t^{h} e^{-(r+\ell) t}
\end{aligned}
$$

Ainsi, en se souvenant que $U$ est analytique en 0 , pour tout $k \geq 1, \int_{0}^{\infty}\left|U^{(2 k)}\right|$ converge. Alors, d'après la proposition 3.1,

$$
\begin{aligned}
\left|R_{m, n}^{U_{\sigma}}(k)\right| & \leq \frac{4}{(2 \pi)^{2 k}} \int_{m}^{n}\left|U_{\sigma}^{(2 k)}(x)\right| d x \\
& =\frac{4}{(2 \pi)^{2 k}} \sigma^{2 k-1} \int_{\sigma m}^{\sigma n}\left|U^{(2 k)}\right| \\
& \leq \frac{4}{(2 \pi)^{2 k}} \sigma^{2 k-1} \int_{0}^{\infty}\left|U^{(2 k)}\right|
\end{aligned}
$$

et par suite, grâce au lemme 3.1 qui entraîne

$$
\sigma=O\left(\frac{1}{\sqrt{n}}\right)
$$

on a,

$$
\sigma R_{m, n}^{U}(k)=O\left(\sigma^{2 k}\right)=O\left(\frac{1}{n^{k}}\right) .
$$

Par ailleurs, on déduit de (3.4) que

$$
\forall j \geq 0 \quad, \quad\left|U^{(j)}(\sigma n)\right| \sim \ell^{j}(\sigma n)^{h} e^{-\ell \sigma n}
$$

quand $n \rightarrow+\infty$, car par le lemme 3.1

$$
\sigma n \geq \frac{\log \Gamma}{\Gamma} \sqrt{n} \rightarrow+\infty
$$

Par suite, grâce à (3.7) et (3.5),

$$
\forall j \geq 0, \quad U^{(j)}(\sigma n)=O\left(n^{\frac{h}{2}} e^{-\ell \sqrt{n} \log \Gamma / \Gamma}\right)
$$

et, en utilisant (3.5) à nouveau, on a

$$
\frac{\sigma}{2} U(\sigma n)+\sum_{j=1}^{k-1} \frac{B_{2 j}}{(2 j) !} \sigma^{2 j} U^{(2 j-1)}(\sigma n)=O\left(\frac{1}{n^{k}}\right) \text {. }
$$

Enfin, comme $h \geq \ell \geq 1$, à l'aide d'une intégration par parties on obtient lorsque $t \rightarrow+\infty$

$$
\int_{t}^{+\infty} u^{h} e^{-\ell u} d u \sim \frac{t^{h} e^{-\ell t}}{\ell}
$$


d'où, en utilisant (3.4) pour $j=0$, lorsque $t \rightarrow+\infty$,

$$
\int_{t}^{+\infty} U=\int_{t}^{+\infty} O\left(u^{h} e^{-\ell u}\right) d u=O\left(\int_{t}^{+\infty} u^{h} e^{-\ell u} d u\right)=O\left(t^{h} e^{-\ell t}\right)
$$

et comme ci-dessus, à l'aide de (3.7) et (3.5),

$$
\int_{\sigma n}^{+\infty} U=O\left(\frac{1}{n^{k}}\right)
$$

Ainsi (3.3) est démontré en reprenant (3.2), (3.9), (3.8) et (3.6).

\subsection{Estimation précise de $\sigma$.}

Lemme 3.3. Soit $m \leq \Gamma n^{1 / 2}$. Alors, pour tout $\ell \in \mathbb{N}$, il existe des fonctions $p_{1}, p_{2}, \ldots, p_{\ell}$ analytiques sur $\mathbb{R}^{+}$telles que

$$
\sigma=\frac{1}{\sqrt{n}} p_{1}(\lambda)+\frac{1}{n} p_{2}(\lambda)+\cdots+\frac{1}{n^{\frac{\ell}{2}}} p_{\ell}(\lambda)+O\left(\frac{1}{n^{\frac{\ell+1}{2}}}\right) .
$$

avec $\lambda=\frac{m}{\sqrt{n}}$. D'autre part, si $x$ est réel,

$$
p_{1}(x)=\frac{H(x)}{x}
$$

où $H$ est définie en 2.3. En particulier, pour $\ell=1$ on obtient :

$$
\sigma=\frac{1}{m} H(\lambda)+O\left(\frac{1}{n}\right)=\frac{1}{\sqrt{n}} \frac{H(\lambda)}{\lambda}+O\left(\frac{1}{n}\right)=\frac{p_{1}(\lambda)}{\sqrt{n}}+O\left(\frac{1}{n}\right) .
$$

Par ailleurs, si $x$ est réel,

$$
p_{2}(x)=\frac{x}{4} \frac{H^{\prime}(x)}{e^{H(x)}-1}=\frac{x}{4} g^{\prime \prime}(x)
$$

où la seconde égalité découle de (2.16).

Démonstration. Le lemme est déja démontré pour $\ell=0$ à l'aide du lemme 3.1 :

$$
\sigma=O\left(\frac{1}{\sqrt{n}}\right) \text {. }
$$

On démontre maintenant (3.10) pour $\ell=1$, puis on verra comment on obtient (3.10) par itération.

Appliquons le lemme 3.2 pour $\ell=h=1: \forall k \geq 1$,

$\sum_{j=m}^{n} \frac{j}{e^{\sigma j}-1}=\frac{1}{\sigma^{2}}\left[\int_{\sigma m}^{+\infty} u+\frac{\sigma}{2} u(\sigma m)-\sum_{j=1}^{k-1} \frac{B_{2 j}}{(2 j) !} \sigma^{2 j} u^{(2 j-1)}(\sigma m)+O\left(\frac{1}{n^{k}}\right)\right]$ où l'on a défini, pour tout $t$ réel,

$$
u(t)=U(1,1, t)=\frac{t}{e^{t}-1} .
$$


Or $n=\sum_{j=m}^{n} \frac{j}{e^{\sigma j}-1}(\operatorname{voir}(1.2))$. En utilisant (2.1), on obtient alors

$$
n=\frac{1}{\sigma^{2}} F(\sigma m)+n R
$$

où $R=1-\frac{1}{n \sigma^{2}} F(\sigma m)$ vérifie

$$
R=\frac{1}{n \sigma^{2}}\left[\frac{\sigma}{2} u(\sigma m)-\sum_{j=1}^{k-1} \frac{B_{2 j}}{(2 j) !} \sigma^{2 j} u^{(2 j-1)}(\sigma m)+O\left(\frac{1}{n^{k}}\right)\right]
$$

pour tout $k \geq 1$, soit, puisque $\frac{1}{\sigma}=O(\sqrt{n})$ grâce au lemme 3.1, pour tout $k \geq 1$

$$
R=\frac{1}{2 n \sigma}\left[u(\sigma m)-2 \sum_{j=1}^{k-1} \frac{B_{2 j}}{(2 j) !} \sigma^{2 j-1} u^{(2 j-1)}(\sigma m)+O\left(\frac{1}{n^{k-\frac{1}{2}}}\right)\right] .
$$

La formule (3.16) appliquée pour $k=1$ donne

$$
R=\frac{1}{2 n \sigma}\left[u(\sigma m)+O\left(\frac{1}{\sqrt{n}}\right)\right]
$$

mais la fonction $u$ est décroissante positive, donc $|u(\sigma m)|=u(\sigma m)$ $\leq u(0)=1$ et par le lemme 3.1 ,

$$
R=O\left(\frac{1}{2 n \sigma}\right)=O\left(\frac{1}{\sqrt{n}}\right),
$$

ce qui entraîne $|R|<1$ pour $n$ assez grand.

La relation (3.15) peut alors s'écrire

$$
\frac{\sigma m}{\sqrt{F(\sigma m)}}=\frac{m}{\sqrt{n} \sqrt{1-R}}
$$

et donc, par la formule (2.8)

$$
G(\sigma m)=\frac{m}{\sqrt{n} \sqrt{1-R}}
$$

et puisque $H=G^{-1}$,

$$
\sigma=\frac{1}{m} H\left(\frac{m}{\sqrt{n}} \frac{1}{\sqrt{1-R}}\right) .
$$

Nous pouvons alors démontrer (3.12) : la formule (3.18) entraîne

$$
\frac{1}{\sqrt{1-R}}=1+O\left(\frac{1}{\sqrt{n}}\right) \text {. }
$$


Or $H^{\prime}$ est analytique en 0 , donc $H^{\prime}$ est bornée au voisinage de 0 , et en appliquant la formule des accroissements finis à $H$ entre $\frac{m}{\sqrt{n}} \frac{1}{\sqrt{1-R}}$ et $\frac{m}{\sqrt{n}}$, on obtient, par (3.20) :

$$
H\left(\frac{m}{\sqrt{n}} \frac{1}{\sqrt{1-R}}\right)=H\left(\frac{m}{\sqrt{n}}\right)+O\left(\frac{m}{n}\right)
$$

ce qui prouve (3.12), à l'aide de la formule (3.19). La formule (3.12) donne la valeur de $p_{1}$ annoncée en (3.11) et démontre (3.10) pour $\ell=1$.

Montrons maintenant comment on obtient $p_{2}$.

Utilisons la notation $\lambda=\frac{m}{\sqrt{n}}$. On reprend la formule (3.19). A l'aide de la première approximation (3.12) de $\sigma$, on va obtenir une meilleure approximation de $R$ à l'aide de (3.16); alors on utilise un développement de Taylor à l'ordre $2 \operatorname{sur}\left(\lambda, \lambda \frac{1}{\sqrt{1-R}}\right)$, cet intervalle étant inclus dans $[0,2 \Gamma]$ pour $n$ assez grand puisque $\lambda \leq \Gamma$ et, d'après (3.20), $\frac{1}{\sqrt{1-R}} \rightarrow 1$ quand $n$ tend vers $+\infty$; ceci est légitime car $H$ est analytique sur $\mathbb{R}^{+}$, et on a alors $H^{\prime \prime}$ bornée sur $[0,2 \Gamma]$ :

$$
H\left(\lambda \frac{1}{\sqrt{1-R}}\right)=H(\lambda)+\lambda\left(\frac{1}{\sqrt{1-R}}-1\right) H^{\prime}(\lambda)+O\left(\lambda^{2}\left(\frac{1}{\sqrt{1-R}}-1\right)^{2}\right)
$$

c'est-à-dire, toujours avec (3.20),

$$
H\left(\lambda \frac{1}{\sqrt{1-R}}\right)=H(\lambda)+\lambda\left(\frac{1}{\sqrt{1-R}}-1\right) H^{\prime}(\lambda)+O\left(\frac{\lambda^{2}}{n}\right)
$$

et ainsi, en reprenant (3.19),

$$
\begin{aligned}
\sigma & =\frac{1}{m} H(\lambda)+\frac{1}{\sqrt{n}}\left(\frac{1}{\sqrt{1-R}}-1\right) H^{\prime}(\lambda)+O\left(\frac{\lambda}{n^{3 / 2}}\right) \\
& =\frac{1}{m} H(\lambda)+\frac{1}{\sqrt{n}}\left(\frac{1}{\sqrt{1-R}}-1\right) H^{\prime}(\lambda)+O\left(\frac{1}{n^{3 / 2}}\right)
\end{aligned}
$$

ce qui procure une meilleure approximation de $\sigma$.

De façon plus précise, d'après (3.17), (3.11) et (3.12) maintenant démontrées,

$$
R=\frac{u(\sigma m)+O\left(\frac{1}{n^{1 / 2}}\right)}{2 n \sigma}=\frac{1}{2 \sqrt{n}} \frac{u(\sigma m)+O\left(\frac{1}{n^{1 / 2}}\right)}{p_{1}(\lambda)+O\left(\frac{1}{n^{1 / 2}}\right)} .
$$

Or par (3.19), (3.20), et le théorème des accroissements finis appliqué à la fonction $u \circ H$ sachant que $u \circ H$ est de classe $C^{1}$ sur $[0,2 \Gamma]$ et que 
$(u \circ H)^{\prime}=H^{\prime} \times u^{\prime} \circ H$ est bornée sur $[0,2 \Gamma]$, on obtient

$$
\begin{aligned}
u(\sigma m)=u\left(H\left(\lambda \frac{1}{\sqrt{1-R}}\right)\right) & =u(H(\lambda))+O\left(\lambda \frac{1}{\sqrt{n}}\right) \\
& =u(H(\lambda))+O\left(\frac{1}{\sqrt{n}}\right) .
\end{aligned}
$$

D'après la relation (2.11), $H(\lambda)$ et $\lambda$ étant de même signe, on a $\frac{H(\lambda)}{\lambda}=$ $\sqrt{F(H(\lambda)}$. Comme $F$ est décroissante et $H$ croissante, $F \circ H$ est décroissante, et pour $0 \leq \lambda \leq 2 \Gamma$, on a

$$
p_{1}(\lambda)=\frac{H(\lambda)}{\lambda} \geq \sqrt{F(H(2 \Gamma))}>0 .
$$

De même, comme $u$ est décroissante, $u(H(\lambda)) \geq u(H(2 \Gamma))>0$. On obtient alors de (3.22) et (3.23)

$$
R=\frac{1}{2 \sqrt{n}} \frac{u \circ H(\lambda)}{p_{1}(\lambda)} \frac{1+(u \circ H(\lambda))^{-1} O\left(\frac{1}{\sqrt{n}}\right)}{1+\left(p_{1}(\lambda)\right)^{-1} O\left(\frac{1}{\sqrt{n}}\right)}
$$

et donc,

$$
R=\frac{1}{2 \sqrt{n}} \frac{u \circ H(\lambda)}{p_{1}(\lambda)}\left(1+O\left(\frac{1}{\sqrt{n}}\right)\right)=\frac{1}{2 \sqrt{n}} \frac{u \circ H(\lambda)}{p_{1}(\lambda)}+O\left(\frac{1}{n}\right) .
$$

Ainsi

Alors, en utilisant (3.18),

$$
R \sim \frac{1}{2 \sqrt{n}} \frac{u \circ H(\lambda)}{p_{1}(\lambda)} .
$$

$\frac{1}{\sqrt{1-R}}-1=(1-R)^{-1 / 2}-1=\frac{1}{2} R+O\left(R^{2}\right)=\frac{1}{4 \sqrt{n}} \frac{u \circ H(\lambda)}{p_{1}(\lambda)}+O\left(\frac{1}{n}\right)$.

D'où, en reprenant (3.21),

$$
\sigma=\frac{1}{\sqrt{n}} p_{1}(\lambda)+\frac{1}{n} \frac{u \circ H(\lambda)}{4 p_{1}(\lambda)} H^{\prime}(\lambda)+O\left(\frac{1}{n^{3 / 2}}\right) .
$$

On retrouve ainsi la formule (3.13) attendue donnant $p_{2}$, avec $p_{2}$ analytique sur $\mathbb{R}^{+}$puisque $u \circ H, H$ et $p_{1}$ le sont et $p_{1}(0)=c$.

De la même façon, on reprend $R$ à l'aide de (3.16) qu'on approche mieux grâce à la nouvelle approximation de $\sigma$; on pousse le développement de Taylor à l'ordre suivant, celui de $\frac{1}{\sqrt{1-R}}$ également, et on obtient, avec la formule d'itération (3.19), $\sigma$ à l'ordre $O\left(\frac{1}{n^{2}}\right)$.

Comme $H$ est analytique sur $\mathbb{R}^{+}$, on peut pousser le développement de Taylor de $H\left(\lambda \frac{1}{\sqrt{1-R}}\right)$ aussi loin que l'on veut, et obtenir ainsi (3.10) pour tout $\ell \in \mathbb{N}^{*}$. 
3.5. Estimation précise de $-\log B$.

Lemme 3.4. On a pour tout $\ell \geq 0$, uniformément pour $m \leq \Gamma n^{\frac{1}{2}}$,

$$
\begin{array}{r}
-\log B=\frac{3}{2} \log \sigma-\frac{1}{2} \log \frac{2 \pi^{2}}{3}-\frac{1}{2} \log \left(1-\frac{3}{\pi^{2}} \int_{0}^{\sigma m} \frac{x^{2} e^{x}}{\left(e^{x}-1\right)^{2}} d x\right) \\
+\sigma r_{1}(\sigma m)+\cdots+\sigma^{\ell} r_{\ell}(\sigma m)+O\left(\frac{1}{n^{\frac{\ell+1}{2}}}\right)
\end{array}
$$

où $r_{1}, \cdots r_{\ell}$ désignent des fonctions analytiques sur $\mathbb{R}^{+}$. En particulier $\grave{a}$ l'ordre $\frac{1}{\sqrt{n}}(\ell=0)$, nous avons :

$$
\begin{aligned}
-\log B=\frac{3}{2} \log \sigma & -\frac{1}{2} \log \frac{2 \pi^{2}}{3} \\
& -\frac{1}{2} \log \left(1-\frac{3}{\pi^{2}} \int_{0}^{\sigma m} \frac{x^{2} e^{x}}{\left(e^{x}-1\right)^{2}} d x\right)+O\left(\frac{1}{\sqrt{n}}\right) .
\end{aligned}
$$

Remarque. La formule (3.26) du lemme 3.4 entraîne, quand $n \rightarrow+\infty$,

$$
B^{2} \sim \sigma^{-3} \frac{2 \pi^{2}}{3}\left(1-\frac{3}{\pi^{2}} \int_{0}^{\sigma m} \frac{x^{2} e^{x}}{\left(e^{x}-1\right)^{2}} d x\right) ;
$$

donc pour tout $\lambda$ fixé, $\lambda \leq \Gamma$, par la formule (3.12) procurant l'équivalent de $\sigma$ et par le théorème des accroissements finis, on obtient,

$$
B^{2} \sim\left(\frac{H(\lambda)}{\lambda \sqrt{n}}\right)^{-3} \frac{2 \pi^{2}}{3}\left(1-\frac{3}{\pi^{2}} \int_{0}^{H(\lambda)} \frac{x^{2} e^{x}}{\left(e^{x}-1\right)^{2}} d x\right) .
$$

Ainsi, lorsque $\lambda \rightarrow 0$, on obtient, puisque $H(\lambda) \sim c \lambda$,

$$
B^{2} \sim \frac{2 \pi^{2}}{3 c^{3}} n^{3 / 2}=\frac{4 \sqrt{6}}{\pi} n^{3 / 2} .
$$

Notons que la formule (3.25) associée au lemme 3.3 donnant l'estimation précise de $\sigma$, nous procure un développement asymptotique de $-\log B$ en fonction de $m$ et $n$.

Démonstration du Lemme 3.4. Partons de la formule (1.3),

$$
B^{2}=2 \sum_{j=m}^{n} \frac{j^{2} e^{\sigma j}}{\left(e^{\sigma j}-1\right)^{2}}=2 A(2,1)+2 A(2,2) .
$$

On applique alors le lemme 3.2 pour $A(2,1)$ et $A(2,2)$ : pour tout $k \geq 1$,

$$
B^{2}=\frac{1}{\sigma^{3}}\left[\int_{\sigma m}^{\infty} U_{2}+\frac{\sigma}{2} U_{2}(\sigma m)-\sum_{j=1}^{k-1} \frac{B_{2 j}}{(2 j) !} \sigma^{2 j} U_{2}^{(2 j-1)}(\sigma m)+O\left(\frac{1}{n^{k}}\right)\right]
$$


où, pour tout $t$ réel,

$$
U_{2}(t)=2 U(2,1, t)+2 U(2,2, t)=\frac{2 t^{2} e^{t}}{\left(e^{t}-1\right)^{2}}
$$

Alors

$$
-\log B=\frac{3}{2} \log \sigma-\frac{1}{2} \log \frac{2 \pi^{2}}{3}-\frac{1}{2} \log \left(\frac{3}{2 \pi^{2}} \int_{\sigma m}^{\infty} U_{2}\right)-\frac{1}{2} \log (1-X)
$$

où $X=\sigma q_{1}(\sigma m)+\sigma^{2} q_{2}(\sigma m)+\sigma^{4} q_{3}(\sigma m)+\cdots+\sigma^{2 k-2} q_{k}(\sigma m)+O\left(\frac{1}{n^{k}}\right)$ avec, si $x$ est réel,

$$
q_{1}(x)=-\frac{1}{\int_{x}^{+\infty} U_{2}} \frac{U_{2}(x)}{2}
$$

et si $i \geq 2$,

$$
q_{i}(x)=\frac{1}{\int_{x}^{+\infty} U_{2}} \frac{B_{2 i-2}}{(2 i-2) !} U_{2}^{(2 i-3)}(x)
$$

et en utilisant le fait que, $U_{2}$ étant positive et $\sigma m \leq c \lambda \leq c \Gamma$ d'après le lemme 3.1 , on a

$$
\int_{\sigma m}^{+\infty} U_{2} \geq \int_{c \Gamma}^{+\infty} U_{2}>0
$$

Notons que, $U_{2}$ étant analytique sur $\mathbb{R}^{+}$, les fonctions $q_{1}, q_{2}, \ldots, q_{k}$ le sont, puisque $U_{2}$ est positive sur $\mathbb{R}$. Notons aussi que $X=O(\sigma)=O\left(n^{-1 / 2}\right)$ quand $n \rightarrow+\infty$. Rappelons alors que, d'un point de vue séries formelles, si $X$ s'écrit $X=\sum_{\gamma \geq 1} a_{\gamma} \sigma^{\gamma}$, alors $-\log (1-X)=\sum_{\gamma \geq 1} b_{\gamma} \sigma^{\gamma}$ avec

$$
b_{\gamma}=\sum_{\substack{i \geq 1 \\ \gamma_{1}+. .+\gamma_{i}=\gamma}} \frac{1}{i} a_{\gamma_{1}} a_{\gamma_{2}} . . a_{\gamma_{i}} \text {. }
$$

Il s'en suit le développement asymptotique de $-\frac{1}{2} \log (1-X)$ :

$$
-\frac{1}{2} \log (1-X)=\sigma r_{1}(\sigma m)+\cdots+\sigma^{2 k-1} r_{2 k-1}(\sigma m)+O\left(\frac{1}{n^{k}}\right)
$$

avec $r_{1}=\frac{1}{2} q_{1}, r_{2}=\frac{1}{4} q_{1}^{2}+\frac{1}{2} q_{2}, r_{3}=\frac{1}{6} q_{1}^{3}+\frac{1}{2} q_{1} q_{2}, \ldots$ des fonctions analytiques sur $\mathbb{R}^{+}$.

On obtient alors le développement asymptotique de $-\log B$ attendu en choisissant $k \geq \frac{\ell+1}{2}$, sachant que

$$
\int_{0}^{+\infty} U_{2}=\frac{2 \pi^{2}}{3}
$$

En effet, on vérifie que $U_{2}=2\left(u-I d . u^{\prime}\right)$ où $u$ est la fonction définie dans la preuve du lemme 3.3, et $I d: x \mapsto x$; donc, grâce à une intégration par 
partie, et avec la formule (2.1), si $x$ est réel,

$$
\begin{aligned}
\int_{x}^{+\infty} U_{2} & =2\left(\int_{x}^{+\infty} u-\int_{x}^{+\infty} I d . u^{\prime}\right) \\
& =2\left(\int_{x}^{+\infty} u-\left\{[I d . u]_{x}^{+\infty}-\int_{x}^{+\infty} u\right\}\right) \\
& =2\left(2 \int_{x}^{+\infty} u+x u(x)\right)=4 F(x)+2 x u(x)
\end{aligned}
$$

et alors il suffit d'utiliser la formule (2.3), $F(0)=\frac{\pi^{2}}{6}$, et de prendre $x=0$ dans (3.29).

\subsection{Estimation précise de $\sum_{j=m}^{n}-\log \left(1-e^{-\sigma j}\right)$.}

Lemme 3.5. Soit

$I(n, m)=\log ((m-1) !)-\log \sqrt{2 \pi}-m(\log m-1)-\frac{1}{2} \log \sigma-\frac{1}{2} \log \frac{1-e^{-\sigma m}}{\sigma m}$.

Définissons $R(n, m)$ par

$$
\sum_{j=m}^{n}-\log \left(1-e^{-\sigma j}\right)=\int_{m}^{+\infty}-\log \left(1-e^{-\sigma x}\right) d x+I(n, m)+R(n, m) .
$$

On a, uniformément pour $m \leq \Gamma n^{\frac{1}{2}}$, et pour tout $\ell \geq 0$,

$$
R(n, m)=-\sum_{i=1}^{\left\lfloor\frac{\ell}{2}\right\rfloor} \sigma^{2 i-1} \frac{B_{2 i}}{(2 i) !} s^{(2 i-1)}(\sigma m)+O\left(\frac{1}{n^{\frac{\ell+1}{2}}}\right),
$$

où $s: x \mapsto-\log \left(\frac{1-e^{-x}}{x}\right)$ est une fonction analytique sur $\mathbb{R}$.

En particulier, pour $\ell=0$,

$$
R(n, m)=O\left(\frac{1}{\sqrt{n}}\right) .
$$

Démonstration. On a

$$
\begin{array}{r}
\sum_{j=m}^{n}-\log \left(1-e^{-\sigma j}\right)=\sum_{j=m}^{n}-\log \frac{1-e^{-\sigma j}}{\sigma j}+\log ((m-1) !) \\
-\log (n !)-(n-m+1) \log \sigma .
\end{array}
$$

Or,

$$
\sum_{j=m}^{n}-\log \frac{1-e^{-\sigma j}}{\sigma j}=\sum_{j=m}^{n} s_{\sigma}(j)
$$


où $s_{\sigma}=s \circ \varphi$ et $s(x)=-\log \left(\frac{1-e^{-x}}{x}\right)$. Par la formule sommatoire d'Euler-MacLaurin, on obtient, pour tout $k \geq 1$

$$
\begin{aligned}
\sum_{j=m}^{n} s_{\sigma}(j)=\int_{m}^{n} s_{\sigma}+ & \frac{1}{2}[ \\
& \left.s_{\sigma}(m)+s_{\sigma}(n)\right] \\
& +\sum_{j=1}^{k-1} \frac{B_{2 j}}{(2 j) !}\left[s_{\sigma}^{(2 j-1)}(n)-s_{\sigma}^{(2 j-1)}(m)\right]+R_{m, n}^{s_{\sigma}}(k)
\end{aligned}
$$

où

$$
\left|R_{m, n}^{s_{\sigma}}(k)\right| \leq \frac{4}{(2 \pi)^{2 k}} \int_{m}^{n}\left|s_{\sigma}^{(2 k)}\right| .
$$

Or,

(i)

$$
\begin{aligned}
\int_{m}^{n} s_{\sigma}=\int_{m}^{\infty}-\log \left(1-e^{-\sigma x}\right) & d x+n \log \sigma+n(\log n-1) \\
& -m \log \sigma-m(\log m-1)+O\left(\frac{1}{n^{k-\frac{1}{2}}}\right)
\end{aligned}
$$

car $s_{\sigma}(x)=-\log \left(1-e^{-\sigma x}\right)+\log \sigma+\log x$ et

$$
\begin{aligned}
\int_{n}^{\infty}-\log \left(1-e^{-\sigma x}\right) d x=\frac{1}{\sigma} \int_{\sigma n}^{\infty}-\log \left(1-e^{-x}\right) d x & =O\left(\frac{1}{\sigma} \int_{\sigma n}^{\infty} e^{-x} d x\right) \\
& =O\left(\frac{1}{\sigma} e^{-\sigma n}\right)
\end{aligned}
$$

soit, par le lemme 3.1,

$$
\int_{n}^{\infty}-\log \left(1-e^{-\sigma x}\right) d x=O\left(\sqrt{n} e^{-\frac{\log \Gamma}{\Gamma} \sqrt{n}}\right)=O\left(\frac{1}{n^{k-\frac{1}{2}}}\right) .
$$

(ii)

$$
\begin{aligned}
s(\sigma n)=-\log \left(1-e^{-\sigma n}\right)+\log (\sigma n) & =\log \sigma+\log n+O\left(e^{-\sigma n}\right) \\
& =\log \sigma+\log n+O\left(\frac{1}{n^{k-\frac{1}{2}}}\right)
\end{aligned}
$$

car à nouveau, par le lemme 3.1,

$$
e^{-\sigma n} \leq e^{-\frac{\log \Gamma}{\Gamma} \sqrt{n}}=O\left(\frac{1}{n^{k-\frac{1}{2}}}\right)
$$

(iii)

$$
s_{\sigma}^{(j)}(n)=\frac{(-1)^{j}(j-1) !}{n^{j}}+O\left(\sigma^{j} e^{-\sigma n}\right)=\frac{(-1)^{j}(j-1) !}{n^{j}}+O\left(\frac{1}{n^{k-\frac{1}{2}}}\right)
$$


car $s^{\prime}(x)=\frac{1}{x}-\frac{e^{-x}}{\left(1-e^{-x}\right)}=\frac{1}{x}-\sum_{\gamma \geq 1} e^{-\gamma x}$ et on obtient par récurrence sur $j \geq 1$ que

$$
\forall x>0 \quad, \quad s^{(j)}(x)=\frac{(-1)^{j}(j-1) !}{x^{j}}+(-1)^{j} \sum_{\gamma \geq 1} \gamma^{j-1} e^{-\gamma x} .
$$

Ainsi $\quad s^{(j)}(x)-\frac{(-1)^{j}(j-1) !}{x^{j}} \sim(-1)^{j} e^{-x}$ quand $x \rightarrow+\infty$, ce qui prouve l'égalité de (iii) puisque $s_{\sigma}^{(j)}(n)=\sigma^{j} s^{(j)}(\sigma n)$.

(iv) $\forall j \geq 2, \int_{0}^{\infty}\left|s^{(j)}\right|$ converge car $s^{(j)}$ est analytique en 0 (car $s$ l'est), et $s^{(j)}(x) \sim \frac{(-1)^{j-1}(j-1) !}{x^{j}}$ quand $x \rightarrow+\infty$.

Donc $\left|R_{m, n}^{s_{\sigma}}(k)\right|=O\left(\sigma^{2 k-1}\right)=O\left(\frac{1}{n^{k-\frac{1}{2}}}\right)$.

Ainsi, en regroupant tous ces résultats, on obtient exactement l'énoncé du lemme, à ceci près que

$$
\begin{aligned}
R(n, m)=-\log \frac{n !}{\sqrt{2 \pi n}\left(\frac{n}{e}\right)^{n}} & -\sum_{j=1}^{k-1} \frac{B_{2 j}}{(2 j) !} \frac{(2 j-2) !}{n^{2 j-1}} \\
& -\sum_{j=1}^{k-1} \frac{B_{2 j}}{(2 j) !} \sigma^{2 j-1} s^{(2 j-1)}(\sigma m)+O\left(\frac{1}{n^{k-\frac{1}{2}}}\right) .
\end{aligned}
$$

Or on connait la formule de Stirling qui peut se déduire de la formule sommatoire d'Euler-Maclaurin appliquée à la fonction log sur $[1, n]$ à l'ordre $k$ (voir [5], chapitre XIII) :

$$
\log \frac{n !}{\sqrt{2 \pi n}\left(\frac{n}{e}\right)^{n}}=\sum_{j=1}^{k-1} \frac{B_{2 j}}{(2 j)(2 j-1)} \frac{1}{n^{2 j-1}}+O\left(\frac{1}{n^{2 k-1}}\right) .
$$

On obtient alors bien le reste $R(n, m)$ voulu en choisissant $k=\left[\frac{\ell}{2}\right]+1$, et par suite le lemme.

\subsection{Estimation de $\log Q$.}

Lemme 3.6. On a, uniformément pour $m \leq \Gamma n^{\frac{1}{2}}$, et pour tout $\ell \geq 0$,

$$
\log Q=\sigma t_{1}(\sigma m)+\sigma^{2} t_{2}(\sigma m)+\cdots+\sigma^{\ell} t_{\ell}(\sigma m)+O\left(\frac{1}{n^{\frac{\ell+1}{2}}}\right),
$$

où $t_{1}, t_{2}, \ldots, t_{\ell}$ sont des fonctions analytiques sur $\mathbb{R}^{+}$. En particulier, pour $\ell=0$,

$$
\log Q=O\left(\frac{1}{\sqrt{n}}\right)
$$


Démonstration. Soit $\ell \geq 0$. Choisissons $k=2 \ell+4$ dans le théorème 1.1 et reprenons la formule (1.4) définissant $Q$. On a alors

$$
E=O\left(n^{-\frac{2 \ell+3}{4}}(\log n)^{\frac{3}{2}(2 \ell+3)}\right)=O\left(n^{-\frac{\ell+1}{2}}\right)
$$

et

$$
Q=1+\sum_{2 \leq i \leq(3 k-6) / 2}(-1)^{i} Q_{2 i}+E,
$$

avec, pour tout $i \geq 2, Q_{2 i}$ combinaisons linéaires de termes de la forme $L_{2}^{-i} L_{h_{1}} L_{h_{2}} \ldots L_{h_{t}}$ avec

$$
h_{1}+h_{2}+\cdots+h_{t}=2 i \text { et } h_{j} \geq 3 \text { pour tout } j=1, \ldots, t
$$

où $L_{h}=\sum_{i=1}^{h} d(h, i) A(h, i)$ est défini dans le théorème 1.1. Or, par le lemme 3.2 , pour tout $k \geq 1$,

$$
L_{h}=\frac{1}{\sigma^{h+1}}\left[\int_{\sigma m}^{+\infty} U_{h}+\frac{\sigma}{2} U_{h}(\sigma m)-\sum_{j=1}^{k-1} \frac{B_{2 j}}{(2 j) !} \sigma^{2 j} U_{h}^{(2 j-1)}(\sigma m)+O\left(\frac{1}{n^{k}}\right)\right],
$$

avec $U_{h}: t \mapsto \sum_{i=1}^{h} d(h, i) U(h, i, t)$ analytique sur $\mathbb{R}^{+}$. Ainsi, avec la formule (3.5) et en utilisant $\sigma m \leq c \frac{m}{\sqrt{n}} \leq c \Gamma$ provenant du lemme 3.1, on obtient, en choisissant $k \geq \frac{\ell+1}{2}$,

$$
L_{h}=\frac{1}{\sigma^{h+1}}\left[\int_{\sigma m}^{+\infty} U_{h}+\sigma f_{h, 1}(\sigma m) \cdots+\sigma^{\ell} f_{h, \ell}(\sigma m)+O\left(\frac{1}{n^{\frac{\ell+1}{2}}}\right)\right]
$$

avec chaque fonction $f_{h, j}$ analytique sur $\mathbb{R}^{+}$.

Posons

$$
\begin{aligned}
E_{\ell}=\left\{x_{0}(\sigma m)+\sigma x_{1}(\sigma m)+\cdots+\right. & \sigma^{\ell} x_{\ell}(\sigma m)+O\left(\frac{1}{n^{\frac{\ell+1}{2}}}\right) \\
& \left.x_{0}, x_{1}, \ldots, x_{\ell} \text { analytiques sur } \mathbb{R}^{+}\right\},
\end{aligned}
$$

et pour $x$ analytique sur $\mathbb{R}^{+}$, le sous-ensemble suivant de $E_{\ell}$,

$$
\begin{aligned}
E_{\ell, x}=\left\{x(\sigma m)+\sigma x_{1}(\sigma m)+\cdots+\sigma^{\ell} x_{\ell}(\sigma m)\right. & +O\left(\frac{1}{n^{\frac{\ell+1}{2}}}\right) ; \\
& \left.x_{1}, \ldots, x_{\ell} \text { analytiques sur } \mathbb{R}^{+}\right\} .
\end{aligned}
$$

Donnons quelques propriétés évidentes de ces ensembles :

(i) pour tout $i \geq 0, \sigma^{i} E_{\ell} \subset E_{\ell}$, c'est-à-dire $\forall u \in E_{\ell}, \sigma^{i} u \in E_{\ell}$,

(ii) $E_{\ell}$ est stable par produit et combinaison linéaire,

(iii) si $\frac{1}{x}$ est analytique sur $\mathbb{R}^{+}$, alors $\forall u \in E_{\ell, x}, \frac{1}{u} \in E_{\ell, \frac{1}{x}}$,

(iv) si $u \in E_{\ell, 1}$, alors $\log u \in E_{\ell, 0}$. 
Rappelons enfin que $L_{2}=B^{2}$ (voir (1.3)), et que par (3.32) et (3.29) on a $\sigma^{3} L_{2} \in E_{\ell, f_{2}}$, avec

$f_{2}(t)=\int_{t}^{+\infty} \frac{2 \gamma^{2} e^{\gamma}}{\left(e^{\gamma}-1\right)^{2}} d \gamma=\int_{t}^{+\infty} U_{2}=4 F(t)+2 t u(t)$ si $t$ est réel, et $\frac{1}{f_{2}}$ analytique sur $\mathbb{R}^{+}$. Donc, par (iii) $\left(\sigma^{3} L_{2}\right)^{-1} \in E_{\ell} ;$ par ailleurs $\sigma^{h+1} L_{h} \in$ $E_{\ell}$ par (3.32). Ainsi $u=\left(\sigma^{3} L_{2}\right)^{-i}\left(\sigma^{h_{1}+1} L_{h_{1}}\right) \ldots\left(\sigma^{h_{t}+1} L_{h_{t}}\right) \in E_{\ell}$ grâce à (ii). En utilisant alors (3.31), on obtient $L_{2}^{-i} L_{h_{1}} \ldots L_{h_{t}}=\sigma^{i-t} u$, avec $2 i \geq 3 t$ et donc $i-t \geq \frac{i}{3}>0$. Finalement, puisque $i-t$ est entier, on a $i-t-1 \geq 0$ et $L_{2}^{-i} L_{h_{1}} \ldots L_{h_{t}}=\sigma v$ avec $v=\sigma^{i-t-1} u \in E_{\ell}$ par (i). Par conséquent, $Q_{2 i}$ étant combinaison linéaire de termes de la forme $L_{2}^{-i} L_{h_{1}} \ldots L_{h_{t}}$, on a $Q_{2 i} \in \sigma E_{\ell} \subset E_{\ell, 0}$ et donc par (1.4) et (3.30), $Q \in E_{\ell, 1}$, et par (iv) $\log Q \in E_{\ell, 0}$, ce qui achève la démonstration du lemme.

\subsection{Démonstration du théorème 1.2 .}

Soit $\ell \geq 0$. A l'aide des lemmes $3.4,3.5$ et 3.6 appliqués à ce même $\ell$, la formule (3.1) devient

$$
\begin{aligned}
& \log r(n, m)=-\frac{1}{2} \log \frac{4 \pi^{4}}{3}+\log \sigma+\log ((m-1) !)-m(\log m-1)+\sigma n \\
&+\int_{m}^{+\infty}-\log \left(1-e^{-\sigma x}\right) d x-\frac{1}{2} \log \left(1-\frac{3}{\pi^{2}} \int_{0}^{\sigma m} \frac{x^{2} e^{x}}{\left(e^{x}-1\right)^{2}} d x\right) \\
&+\sigma z_{1}(\sigma m)+\sigma^{2} z_{2}(\sigma m)+\cdots+\sigma^{\ell} z_{\ell}(\sigma m)+O\left(\frac{1}{n^{\frac{\ell+1}{2}}}\right)
\end{aligned}
$$

où $z_{j}=r_{j}+\tilde{s}_{j}+t_{j}$ si $j=1,2, \ldots, \ell$, avec

$\tilde{s}_{j}=\left\{\begin{array}{ll}-\frac{B_{2 i}}{(2 i) !} s^{(2 i-1)} & \text { si } j=2 i-1 \\ 0 & \text { si } j \text { est pair }\end{array} ;\right.$ ainsi chaque fonction $z_{j}$ est analytique sur $\mathbb{R}^{+}$. En utilisant la valeur $c=\frac{\pi}{\sqrt{6}}$, on peut écrire, après simplification des calculs, et en se souvenant que $\lambda=\frac{m}{\sqrt{n}}$,

$$
\begin{aligned}
\log r(n, m)= & \log \left(\frac{e^{2 c \sqrt{n}}}{4 \sqrt{3} n}\left(\frac{c}{\sqrt{n}}\right)^{m-1}(m-1) !\right) \\
& -\sqrt{n} v_{0}(\lambda)+\sqrt{n} v(\sigma m)+w(\sigma m) \\
& +\sigma z_{1}(\sigma m)+\sigma^{2} z_{2}(\sigma m)+\cdots+\sigma^{\ell} z_{\ell}(\sigma m)+O\left(\frac{1}{n^{\frac{\ell+1}{2}}}\right)
\end{aligned}
$$


où, pour tout réel $t$,

(3.34) $v_{0}(t)=t \log t+2 c+(\log c-1) t$

$$
\begin{aligned}
& \text { (3.35) } v(t)=\frac{t}{\lambda}+\frac{\lambda}{t} \int_{t}^{+\infty}-\log \left(1-e^{-x}\right) d x=\frac{t}{\lambda}+\lambda \log \left(1-e^{-t}\right)+\frac{\lambda}{t} F(t) \\
& \text { (3.36) } w(t)=\log \frac{t}{\lambda c}-\frac{1}{2} \log \left(\frac{1-e^{-t}}{t}\right)-\frac{1}{2} \log \left(1-\frac{3}{\pi^{2}} \int_{0}^{t} \frac{x^{2} e^{x}}{\left(e^{x}-1\right)^{2}} d x\right)
\end{aligned}
$$

la seconde égalité de (3.35) s'obtenant à la suite d'une intégration par partie et en reprenant la formule (2.1) définissant $F$.

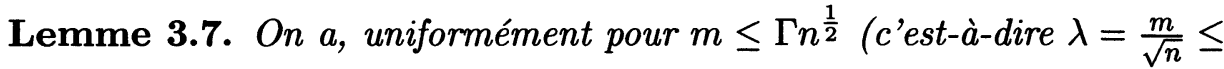
$\Gamma)$, pour tout $\ell \geq 0$, toute fonction $f$ analytique sur $\mathbb{R}^{+}, \tilde{f}$ analytique sur $\mathbb{R}^{+*}$, et tout $i$ vérifiant $1 \leq i \leq \ell$,

$$
f(\sigma m)=f(H(\lambda))+\frac{1}{\sqrt{n}} f_{1}(\lambda)+\cdots+\frac{1}{n^{\frac{\ell}{2}}} f_{\ell}(\lambda)+O\left(\frac{1}{n^{\frac{\ell+1}{2}}}\right),
$$

avec $f_{1}, \ldots, f_{\ell}$ analytiques sur $\mathbb{R}^{+}$, et $f_{1}(\lambda)=\lambda p_{2}(\lambda) f^{\prime}(H(\lambda))$,

$$
\begin{aligned}
\sigma^{i} f(\sigma m)=\frac{1}{n^{\frac{i}{2}}} \frac{H^{i}(\lambda)}{\lambda^{i}} f(H(\lambda))+ & \frac{1}{n^{\frac{i+1}{2}}} f_{i, i+1}(\lambda) \\
& +\cdots+\frac{1}{n^{\frac{\ell}{2}}} f_{i, \ell}(\lambda)+O\left(\frac{1}{n^{\frac{\ell+1}{2}}}\right)
\end{aligned}
$$

avec $f_{i, i+1}, \ldots, f_{i, \ell}$ analytiques sur $\mathbb{R}^{+}$, et

$$
\tilde{f}\left(\frac{\sigma m}{\lambda}\right)=f\left(\frac{H(\lambda)}{\lambda}\right)+\frac{1}{\sqrt{n}} \tilde{f}_{1}(\lambda)+\cdots+\frac{1}{n^{\frac{\ell}{2}}} \tilde{f}_{\ell}(\lambda)+O\left(\frac{1}{n^{\frac{\ell+1}{2}}}\right)
$$

avec $\tilde{f}_{1}, \ldots, \tilde{f}_{\ell}$ analytiques sur $\mathbb{R}^{+}$, et $\tilde{f}_{1}(\lambda)=p_{2}(\lambda) f^{\prime}\left(\frac{H(\lambda)}{\lambda}\right)$.

Démonstration. Soit $\ell \geq 0$. On a alors, d'après la formule (3.10) du lemme 3.3 le développement asymptotique suivant de $\sigma$ :

$$
\sigma=\frac{1}{\sqrt{n}} p_{1}(\lambda)+\frac{1}{n} p_{2}(\lambda)+\cdots+\frac{1}{n^{\frac{\ell+1}{2}}} p_{\ell+1}(\lambda)+O\left(\frac{1}{n^{\frac{\ell+2}{2}}}\right) .
$$

On obtient alors, compte tenu que $\lambda=\frac{m}{\sqrt{n}} \leq \Gamma$, et de (3.11)

$$
\begin{aligned}
& \sigma m=H(\lambda)+\frac{\lambda p_{2}(\lambda)}{\sqrt{n}}+\cdots+\frac{\lambda p_{\ell+1}(\lambda)}{n^{\frac{\ell}{2}}}+O\left(\frac{1}{n^{\frac{\ell+1}{2}}}\right), \\
& \frac{\sigma m}{\lambda}=\frac{H(\lambda)}{\lambda}+\frac{p_{2}(\lambda)}{\sqrt{n}}+\cdots+\frac{p_{\ell}(\lambda)}{n^{\frac{\ell}{2}}}+O\left(\frac{1}{n^{\frac{\ell+1}{2}}}\right) .
\end{aligned}
$$

On en déduit les formules (3.37) et (3.39) en appliquant la formule de Taylor respectivement aux fonctions $f$ et $\tilde{f}$, à l'ordre $\ell+1$, respectivement 
sur $(\sigma m, H(\lambda))$ et $\left(\frac{\sigma m}{\lambda}, \frac{H(\lambda)}{\lambda}\right)$ et en se souvenant que les fonctions $p_{1}=\frac{H}{I d}, p_{2}, \ldots, p_{\ell+1}$ sont analytiques sur $\mathbb{R}^{+}$, que $p_{1}$ vérifie $(3.24)$, et que $\lambda \leq \Gamma$.

La formule (3.38) se déduit, elle, de (3.37) et du développement asymptotique (3.10) de $\sigma$.

Terminons maintenant la démonstration du théorème 1.2.

(i) D'abord, par la formule (3.38) du lemme 3.7, on obtient

$$
\sigma z_{1}(\sigma m)+\cdots+\sigma^{\ell} z_{\ell}(\sigma m)=\frac{g_{1,3}(\lambda)}{\sqrt{n}}+\cdots+\frac{g_{\ell, 3}(\lambda)}{n^{\frac{\ell}{2}}}+O\left(\frac{1}{n^{\frac{\ell+1}{2}}}\right)
$$

avec toutes les fonctions $g_{i, 3}, i=1, \ldots, \ell$ analytiques sur $\mathbb{R}^{+}$.

(ii) Ensuite, en appliquant la formule (3.37) du lemme 3.7 aux fonctions $t \mapsto \log \left(\frac{1-e^{-t}}{t}\right)$ et $t \mapsto \log \left(1-\frac{3}{\pi^{2}} \int_{0}^{t} \frac{x^{2} e^{x}}{\left(e^{x}-1\right)^{2}} d x\right)$, et la formule (3.39) du lemme 3.7 à la fonction $t \mapsto \log \frac{t}{c}$, on obtient

$$
\begin{aligned}
& w(\sigma m)=\log \left(\frac{H(\lambda)}{c \lambda}\right)- \frac{1}{2} \log \left(\frac{1-e^{-H(\lambda)}}{H(\lambda)}\right) \\
&-\frac{1}{2} \log \left(1-\frac{3}{\pi^{2}} \int_{0}^{H(\lambda)} \frac{t^{2} e^{t}}{\left(e^{t}-1\right)^{2}} d t\right) \\
&+\frac{g_{1,2}(\lambda)}{\sqrt{n}}+\cdots+\frac{g_{\ell, 2}(\lambda)}{n^{\frac{\ell}{2}}}+O\left(\frac{1}{n^{\frac{\ell+1}{2}}}\right)
\end{aligned}
$$

avec toutes les fonctions $g_{i, 2}, i=1, \ldots, \ell$ analytiques sur $\mathbb{R}^{+}$. On obtient donc, par (1.10),

$$
w(\sigma m)=g_{0}(\lambda)+\frac{g_{1,2}(\lambda)}{\sqrt{n}}+\cdots+\frac{g_{\ell, 2}(\lambda)}{n^{\frac{\ell}{2}}}+O\left(\frac{1}{n^{\frac{\ell+1}{2}}}\right),
$$

(iii) Enfin, si l'on reprend la fonction $g$ définie dans la formule (2.12) du paragraphe $\mathbf{2 . 4}$, on a

$$
\sqrt{n} v(\sigma m)=\sqrt{n} g(\lambda)+\frac{g_{1,1}(\lambda)}{\sqrt{n}}+\cdots+\frac{g_{\ell, 1}(\lambda)}{n^{\frac{\ell}{2}}}+O\left(\frac{1}{n^{\frac{\ell+1}{2}}}\right),
$$

avec toutes les fonctions $g_{i, 1}, i=1, \ldots, \ell$ analytiques sur $\mathbb{R}^{+}$.

Pour démontrer cela appliquons la formule de Taylor à la fonction $v$ sur 
l'intervalle $(\sigma m, H(\lambda)) \subset \mathbb{R}^{+*}:$ il existe $d$ élément de $(\sigma m, H(\lambda))$ tel que

$$
\begin{gathered}
v(\sigma m)=v(H(\lambda))+(\sigma m-H(\lambda)) v^{\prime}(H(\lambda)) \\
+\frac{(\sigma m-H(\lambda))^{2}}{2} v^{\prime \prime}(H(\lambda))+\cdots \\
+\frac{(\sigma m-H(\lambda))^{\ell+1}}{(\ell+1) !} v^{(\ell+1)}(H(\lambda))+\frac{(\sigma m-H(\lambda))^{\ell+2}}{(\ell+2) !} v^{(\ell+2)}(d) .
\end{gathered}
$$

Notons également que si l'on dérive la seconde égalité de (3.35) on obtient, pour tout $t$ réel,

$$
v^{\prime}(t)=\frac{1}{\lambda}-\lambda \frac{F(t)}{t^{2}}
$$

et par conséquent, par récurrence sur $i \geq 2$, l'existence de $X_{i}$ analytique sur $\mathbb{R}^{+}$, telle que pour $t>0$,

$$
v^{(i)}(t)=\lambda \frac{X_{i}(t)}{t^{i+1}}
$$

Or, d'une part, l'expression (2.13) de $g$ donne immédiatement, à l'aide de la première égalité de (3.35),

$$
v(H(\lambda))=g(\lambda) .
$$

D'autre part, d'après (3.45) et (2.11),

$$
v^{\prime}(H(\lambda))=0 \text {. }
$$

Or, à l'aide de (3.46) et (3.40), pour $2 \leq i \leq \ell+1$,

$$
\frac{(\sigma m-H(\lambda))^{i}}{(i) !} v^{(i)}(H(\lambda))=\frac{X_{i, i}(\lambda)}{n^{\frac{i}{2}}}+\cdots+\frac{X_{i, \ell+1}(\lambda)}{n^{\frac{\ell+1}{2}}}+O\left(\frac{1}{n^{\frac{\ell+2}{2}}}\right)
$$

avec $X_{i, i}, \ldots, X_{i, \ell+1}$ analytiques sur $\mathbb{R}^{+}$, et $X_{i, i}=\frac{p_{2}^{i} X_{i} \circ H}{i ! p_{1}^{i+1}}$.

De plus $d \sim H(\lambda)$ quand $n$ tend vers $+\infty$ car $d$ est compris entre $\sigma m$ et $H(\lambda)$ et $\sigma m \sim H(\lambda)$ : en effet, par (3.40), (3.11) et (3.24)

$$
\frac{\sigma m}{H(\lambda)}=1+\frac{1}{H(\lambda)} O\left(\frac{\lambda}{\sqrt{n}}\right)=1+O\left(\frac{1}{p_{1}(\lambda) \sqrt{n}}\right)=1+O\left(\frac{1}{\sqrt{n}}\right) .
$$

Donc, en reprenant à nouveau (3.46) et (3.40),

$$
\frac{(\sigma m-H(\lambda))^{\ell+2}}{(\ell+2) !} v^{(\ell+2)}(d)=O\left(\frac{1}{n^{\frac{\ell+2}{2}}}\right) .
$$

On obtient bien alors (3.43) à l'aide de (3.47), (3.48), (3.49) et (3.50).

Finalement, en posant $\tilde{g}=g-v_{0}$ et $g_{i}=g_{i, 1}+g_{i, 2}+g_{i, 3}$ pour $i=1, \ldots, \ell$, on obtient le théorème en reprenant (3.33), (3.34), (3.43), (3.42) et (3.41). 
Pour terminer la démonstration, prouvons la deuxième égalité de (1.10). Par (3.27), (3.28) et (3.29), on a

$1-\frac{3}{\pi^{2}} \int_{0}^{H(x)} \frac{t^{2} e^{t}}{\left(e^{t}-1\right)^{2}} d t=\frac{3}{2 \pi^{2}} \int_{H(x)}^{+\infty} U_{2}=\frac{3}{\pi^{2}}(2 F(H(x))+H(x) u(H(x)))$,

d'où, compte-tenu de la relation (2.11), de la définition (3.14) de $u$, et puisque $\frac{3}{\pi^{2}}=\frac{1}{2 c^{2}}$

$$
\begin{aligned}
1-\frac{3}{\pi^{2}} \int_{0}^{H(x)} \frac{t^{2} e^{t}}{\left(e^{t}-1\right)^{2}} d t & =\frac{3}{\pi^{2}}\left(2\left(\frac{H(x)}{x}\right)^{2}+\frac{H^{2}(x)}{e^{H(x)}-1}\right) \\
& =\frac{H^{2}(x)}{c^{2} x^{2}\left(e^{H(x)}-1\right)}\left(e^{H(x)}-1+\frac{x^{2}}{2}\right),
\end{aligned}
$$

et en reprenant la première égalité de (1.10) démontrée ci-avant,

$$
\begin{aligned}
g_{0}(x) & =-\frac{1}{2} \log \left(\left(\frac{c x}{H(x)}\right)^{2} \frac{1-e^{-H(x)}}{H(x)} \frac{H^{2}(x)}{c^{2} x^{2}\left(e^{H(x)}-1\right)}\left(e^{H(x)}-1+\frac{x^{2}}{2}\right)\right) \\
& =\frac{H(x)}{2}-\frac{1}{2} \log \left(1+\frac{e^{H(x)}-1-H(x)+\frac{x^{2}}{2}}{H(x)}\right)
\end{aligned}
$$

ce qui démontre bien la deuxième égalité de (1.10).

\section{Applications}

D'abord, nous allons voir que l'on retrouve le développement asymptotique de $p(n)=r(n, 1)$, le nombre de partitions de l'entier $n$ en appliquant le théorème 1.2 pour $m=1$. Ensuite nous appliquerons ce même théorème pour $m$ respectivement au voisinage de $n^{1 / 4}, n^{1 / 3}$ et $n^{3 / 8}$, et finalement pour $m=\lambda \sqrt{n}$, ce qui améliorera les résultats $(1.5),(1.8),(1.6)$ et (1.7). Ecrivons les développements en série entière de $\tilde{g}$ et $g_{0}$ :

$$
\tilde{g}(\lambda)=\sum_{n \geq 2} a_{n} \lambda^{n}, g_{0}(\lambda)=\sum_{n \geq 1} b_{n} \lambda^{n}
$$

$a_{2}, a_{3}, \ldots, a_{7}$ étant donnés par (2.14) en 2.4, et le développement en série entière de $H$ donné en (2.10) associé à la définition de $g_{0}$ donné dans le théorème 1.2 nous procure les coefficients $b_{n}$. 
On a par exemple :

$$
\begin{cases}b_{1}=\frac{1}{4} \frac{c^{2}-1}{c} & b_{4}=\frac{c^{8}-50 c^{6}-50 c^{4}-90 c^{2}+45}{5760 c^{4}} \\ b_{2}=-\frac{1}{48} \frac{c^{4}+3}{c^{2}} & b_{5}=\frac{40 c^{8}+765 c^{6}+285 c^{4}+135 c^{2}-81}{69120 c^{5}} \\ b_{3}=\frac{1}{96} \frac{3 c^{4}+1}{c^{3}} & \ldots\end{cases}
$$

Bien entendu, plus $m$ est petit, moins on aura besoin de coefficients. Dans les développements qui suivent, on n'utilise que les coefficients des fonctions $\tilde{g}$ et $g_{0}$ pour obtenir une précision $\frac{1}{\sqrt{n}}$. On a vu comment on pourrait trouver les fonctions $g_{1}, \ldots, g_{\ell-1}$, mais au prix de calculs très couteux; cependant, puisque l'on a prouvé l'existence de ces fonctions, on peut extraire leurs coefficients de Taylor de proche en proche en réinjectant les développements asymptotiques dans la formule récursive $r(n, m)=$ $r(n, m+1)+r(n-m, m)$. Ceci nous permet d'obtenir alors la précision $\frac{1}{(\sqrt{n})^{\ell}}$ pour tout $\ell$. Ceci sera précisé dans $[8]$.

4.1. $p(n)$.

Reprenons le théorème 1.2 avec $m=1$, à l'ordre $\frac{1}{\sqrt{n}}$; on obtient

$$
p(n)=\frac{e^{2 c \sqrt{n}}}{4 \sqrt{3} n}\left[1+O\left(\frac{1}{\sqrt{n}}\right)\right]
$$

formule bien connue depuis Hardy et Ramanujan (voir [4]).

4.2. $r(n, m)$ pour $m \leq n^{\frac{1}{4}}$.

On a alors $\lambda=\frac{m}{\sqrt{n}} \leq \frac{1}{n^{1 / 4}}$. On obtient, en ne conservant que les termes d'ordre de grandeur supérieur ou égal à $n^{-1 / 2}$,

$$
\begin{aligned}
r(n, m)=\frac{e^{2 c \sqrt{n}}}{4 \sqrt{3} n}\left(\frac{\pi}{\sqrt{6 n}}\right)^{m-1}(m-1) ! \exp \left\{\sqrt{n}\left(a_{2} \lambda^{2}+a_{3} \lambda^{3}\right)\right. & \\
\left.+b_{1} \lambda\right\} & {\left[1+O\left(\frac{1}{\sqrt{n}}\right)\right] }
\end{aligned}
$$

formule plus précise que (1.5) (la valeur des coefficients $a_{i}$ et $b_{i}$ est donnée en (2.14) et (4.1)). 
4.3. $r(n, m)$ pour $m \leq n^{\frac{1}{3}}$.

De même que ci-dessus, on obtient la formule suivante pour $r(n, m)$, plus précise que (1.8),

$$
\begin{aligned}
r(n, m)=\frac{e^{2 c \sqrt{n}}}{4 \sqrt{3} n}\left(\frac{\pi}{\sqrt{6 n}}\right)^{m-1}(m-1) ! \exp \left\{\sqrt{n}\left(a_{2} \lambda^{2}+a_{3} \lambda^{3}+a_{4} \lambda^{4}+a_{5} \lambda^{5}\right)\right. \\
\left.+b_{1} \lambda+b_{2} \lambda^{2}\right\}\left[1+O\left(\frac{1}{\sqrt{n}}\right)\right] .
\end{aligned}
$$

4.4. $r(n, m)$ pour $m \leq n^{\frac{3}{8}}$.

Le développement suivant améliore (1.6),

$$
\begin{aligned}
r(n, m) & =\frac{e^{2 c \sqrt{n}}}{4 \sqrt{3} n}\left(\frac{\pi}{\sqrt{6 n}}\right)^{m-1}(m-1) ! \exp \left\{\sqrt { n } \left(a_{2} \lambda^{2}+a_{3} \lambda^{3}+a_{4} \lambda^{4}\right.\right. \\
& \left.\left.+a_{5} \lambda^{5}+a_{6} \lambda^{6}+a_{7} \lambda^{7}\right)+b_{1} \lambda+b_{2} \lambda^{2}+b_{3} \lambda^{3}\right\}\left[1+O\left(\frac{1}{\sqrt{n}}\right)\right] .
\end{aligned}
$$

4.5. $r(n, m)$ pour $m=\lambda \sqrt{n}$.

$$
r(n, \sqrt{n})=\frac{e^{2 c \sqrt{n}}}{4 \sqrt{3} n}\left(\frac{\pi}{\sqrt{6 n}}\right)^{m-1}(m-1) ! \exp \left\{\sqrt{n} \tilde{g}(\lambda)+g_{0}(\lambda)\right\}\left[1+O\left(\frac{1}{\sqrt{n}}\right)\right] .
$$

Le tableau ci-dessous donne quelques valeurs des fonctions $g(\lambda), \tilde{g}(\lambda)$, $g_{0}(\lambda)$ et $H(\lambda)$. Rappelons que $\tilde{g}(\lambda)=g(\lambda)-(\lambda \log \lambda+2 c+(\log c-1) \lambda)$, que toutes ces fonctions s'obtiennent à l'aide de la fonction $H$, dont on peut calculer les valeurs puisque c'est la fonction réciproque de $G$ définie en $\mathbf{2 . 2}$ (on peut utiliser par exemple la méthode de Newton, voir [1]). 


\begin{tabular}{|c|c|c|c|c|}
\hline$\lambda$ & $g(\lambda)$ & $\tilde{\boldsymbol{g}}(\lambda)$ & $\overline{g_{0}(\lambda)}$ & $H(\lambda)$ \\
\hline 0 & $\begin{aligned} & 2 \pi / \sqrt{6} \\
\simeq & 2.56510\end{aligned}$ & 0 & 0 & 0 \\
\hline 0.01 & 2.51148 & -0.00005 & 0.00125 & 0.01276 \\
\hline 0.02 & 2.47163 & -0.00021 & 0.00249 & 0.02545 \\
\hline 0.03 & 2.43691 & -0.00046 & 0.00371 & 0.03803 \\
\hline 0.04 & 2.40548 & -0.00082 & 0.00492 & 0.05052 \\
\hline 0.05 & 2.37648 & -0.00127 & 0.00611 & 0.06291 \\
\hline 0.1 & 2.25471 & -0.00501 & 0.01189 & 0.12350 \\
\hline 0.2 & 2.07345 & -0.01953 & 0.02257 & 0.23836 \\
\hline 0.3 & 1.93573 & -0.04283 & 0.03223 & 0.34571 \\
\hline 0.4 & 1.82381 & -0.07432 & 0.04101 & 0.44647 \\
\hline 0.5 & 1.72951 & -0.11344 & 0.04905 & 0.54139 \\
\hline 0.6 & 1.64818 & -0.15973 & 0.05643 & 0.63112 \\
\hline 0.7 & 1.57685 & -0.21277 & 0.06324 & 0.71620 \\
\hline 0.8 & 1.51349 & -0.27217 & 0.06956 & 0.79708 \\
\hline 0.9 & 1.45663 & -0.33761 & 0.07542 & 0.87416 \\
\hline 1.0 & 1.40519 & -0.40876 & 0.08090 & 0.94778 \\
\hline 1.1 & 1.35831 & -0.48536 & 0.08601 & 1.01824 \\
\hline 1.2 & 1.31535 & -0.56715 & 0.09081 & 1.08580 \\
\hline 1.3 & 1.27577 & -0.65391 & 0.09533 & 1.15069 \\
\hline 1.4 & 1.23914 & -0.74541 & 0.09958 & 1.21312 \\
\hline 1.5 & 1.20511 & -0.84147 & 0.10360 & 1.27327 \\
\hline 1.6 & 1.17337 & -0.94149 & 0.10740 & 1.33129 \\
\hline 2 & 1.06495 & -1.38415 & 0.12078 & 1.54487 \\
\hline 3 & 0.87690 & -2.73059 & 0.14539 & 1.98230 \\
\hline 4 & 0.75378 & -4.35190 & 0.16242 & 2.32809 \\
\hline 5 & 0.66555 & -6.19099 & 0.17508 & 2.61432 \\
\hline 6 & 0.59858 & -8.21018 & 0.18494 & 2.85869 \\
\hline 7 & 0.54567 & -10.38276 & 0.19290 & 3.07201 \\
\hline 8 & 0.50262 & -12.68881 & 0.19949 & 3.26137 \\
\hline 9 & 0.46679 & -15.11298 & 0.20506 & 3.43167 \\
\hline 10 & 0.43641 & -17.64304 & 0.20985 & 3.58644 \\
\hline$+\infty$ & 0 & $-\infty$ & 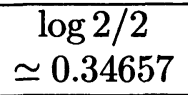 & $+\infty$ \\
\hline
\end{tabular}

\section{Bibliographie}

[1] J. Dixmier, J.L. Nicolas, Partitions sans petits sommants. A tribute to Paul Erdős, 121152. Cambridge Univ. Press, Cambridge, 1990.

[2] J. Dixmier, J.L. Nicolas, Partitions without small parts. Number theory, Vol. I (Budapest, 1987), 9-33, North-Holland, Amsterdam, 1990. 
[3] G. Freiman, J. Pitman, Partitions into distinct large parts. J. Austral. Math. Soc. Ser. A 57(3) (1994), 386-416.

[4] G. H. HARdy, S. Ramanujan, Asymptotic formulae in combinatory analysis. J. London Math. Society 17 (1918), 75-115.

[5] G. H. HaRdy, Divergent series. Oxford, at the Clarendon Press, 1949.

[6] J. HERzoG, Gleichmässige asymptotische Formeln für parameterabhängige Partitionfunktionen. Thèse de l'Université J. F. Goethe, Frankfurt am Main, mars 1987.

[7] D. E. KNUTH, The Art of Computer Programming vol. 2. Addison Wesley, $2^{\text {nd }}$ edition, 1981.

[8] E. MosAKI, Thèse de l'Université Lyon 1 (en préparation).

[9] J.-L. NiCOLAS, A. SÁRKözY, On partitions without small parts. J. de Théorie des Nombres de Bordeaux 12 (2000), 227-254.

[10] G. SZEKERES, An asymptotic formula in the theory of partitions. Quart. J. Math., Oxford 2 (1951), 85-108.

[11] G. SzEKERES, Some asymptotic formulae in the theory of partitions. II. Quart. J. Math., Oxford 4 (1953), 96-111.

Elie Mosaki et Jean-Louis NICOLAS

Université Claude Bernard (Lyon 1)

21 avenue Claude Bernard

F-69622 Villeurbanne Cedex, France

E-mail : mosaki@euler univ-lyon1.fr, jlnicola@in2p3.fr

András SÁRKÖZY

Eötvös Loránd University

Department of Algebra and Number Theory

H-1117 Budapest, Pázmány Péter sétány 1/C, Hungary

E-mail : sarkozy@cs.elte.hu 